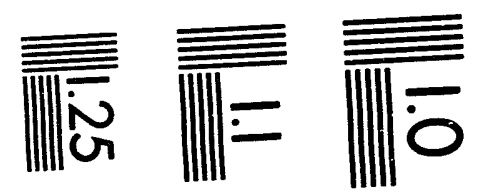

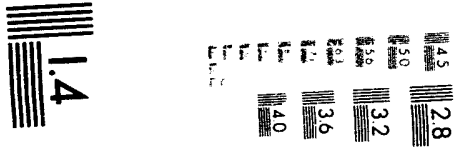

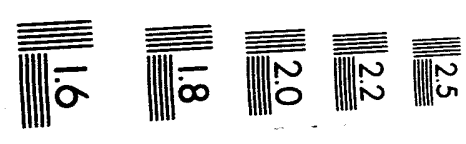



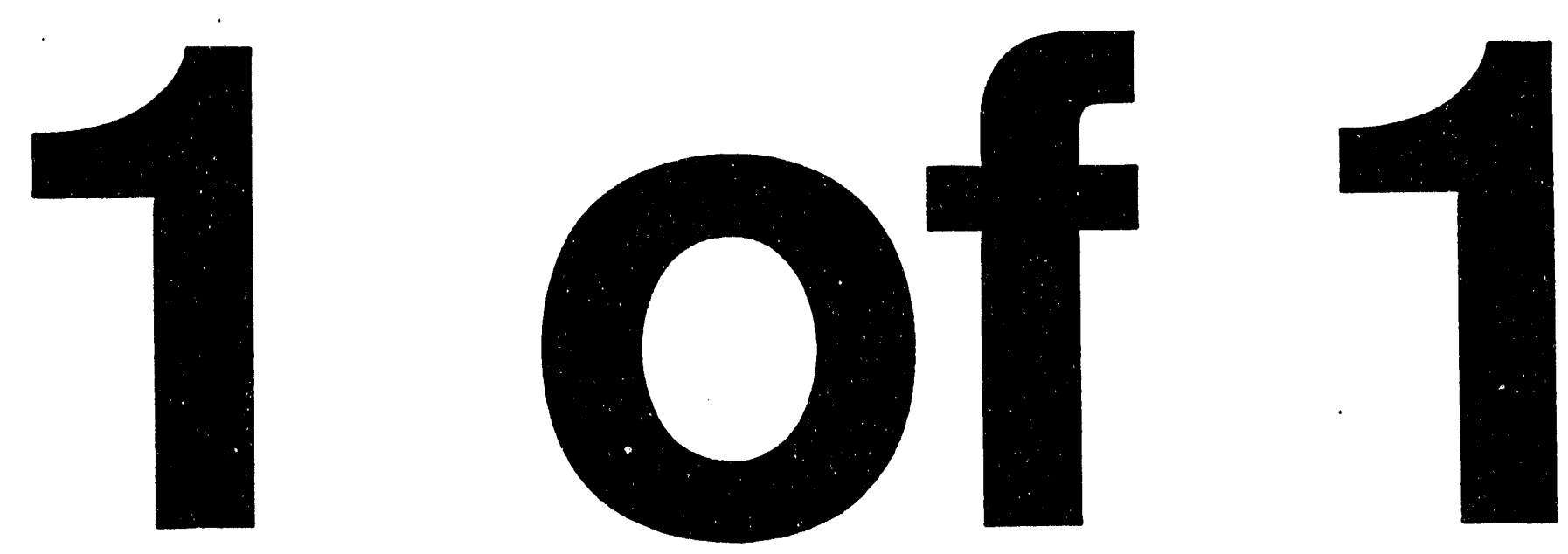


\title{
"Visually Guided Control Systems: A New Generation of System Analysis and Design"
}

\author{
Bijoy Kumar Ghosh * \\ Department of Systems Science and Mathematics \\ Washington University \\ Campus Box 1040 \\ One Brookings Drive \\ St. Louis, MO 63130-4899
}

April 4, 1994

\section{A Brief Summary of Accomplishments}

The main emphasis of the proposal had been to study control systems for which the observation function is perspective. Since a CCD camera observes points upto a perspective projection, it is hoped that these dynamical systems would be useful in the study of visually guided control systems: systems for which the feedback control is to be generated with the aid of a CCD camera. We basically divided our task into three distinct subproblems.

1. To define a perspective dynamical system and study problems in this context that arises as a result of state observability, parameter identifiability and controllability. We also proposed to look into the prospect of applying dynamic observers for on line parameter estimation.

2. To apply the proposed perspective theory in the context of controling a robot arm. The robot is proposed to be controlled with the aid of a pair of CCD cameras for the purpose of dynamic obstacle avoidance and manipulation.

3. The theory and application of a perspective dynamical system in the context of controlling a robot manipulator is to be extended to a general context wherein a visual cognition framework involes image understanding using multiresolution analysis and various other biologically plausible framework and the control task includes visual attention, as a specific choice for active vision.

\footnotetext{
*Proposal No. DE-FG02-90ER14140.
} 
Our main research accomplishments are now detailed.

\subsection{Perspective Observability and Identifiability Problems}

We introduce and study a linear dynamical system with a perspective observation function. The study of these systems has been shown to be useful in motion and shape estimation problems in machine vision. We introduce the notion of perspective observability and obtain as a special case a necessary and sufficient condition that would guarantee observability of the initial condition of the linear dynamical system up to a one parameter magnitude scaling. An earlier result on the problem of observability of a linear dynamical system due to PopovBelevitch-Hautus has been generalized and applied to the problem of observing the initial condition of a linear dynamical system described on the space of $d$ dimensional affine planes in $\mathbb{R}^{n}$. Subsequently a new realization theory is introduced which is useful for studying linear systems with perspective observation. A new rescaling algorithm is described in order to identify parameters of the perspective system. The identification problem is further analyzed in detail for various problems that are of interest in machine vision. In order to obtain recursive filters that would identify the parameters, we consider the problem of estimating the range information of features on an affine plane in $\mathbb{R}^{3}$ by observing its image with the aid of a CCD camera, wherein we assume that the camera is undergoing a known motion. The features considered are points, lines and planar curves located on planar surfaces of static objects. The dynamics of the moving projections of the features on the image plane have been described as a suitable differential equation on an appropriate feature space. This dynamics is used to estimate feature parameters from which the range information is readily available. The proposed identification has been carried out via a newly introduced Identifier Based Observer.

\subsection{Visually Guided Robot Tracking and Grasping of a Moving Object}

We present a new approach to the problem of tracking and grasping of a moving object by a manipulator with multiple cameras. We propose a new object-centered model for the motion of the object by defining a 3-D reference point in the object. since the reference point is used to represent the translational motion of the object, the tracking can be designed with respect to the 3-D point. However, no attempt is made to recover 3-D feature points of the object based on stereo matching. Instead, only the image of the reference point in each camera is needed and determined from the 2-D features of the object within the same camera. By properly defining an eror function, between the image of the gripper and that of the reference point, the image based tracking control law is then obtained using a nonlinear regulator theory.

\subsection{A Multiresolution Framework for Image Segmentation}

The problem of segmentation of a smooth image has been studied using multiresolution analysis. The analysis has been carried out with the aid of a new orthonormal wavelet basis. 
A procedure has been developed to approximate an image at a coarse resolution by dropping its component at finer resolution. The proposed wavelet basis has been used to represent the fine resolution component of the image. An algorithm is proposed which performs an initial segmentation on the coarse approximation of the image and a region information table is formed. The table is used and updated repeatedly via a 'region refinement procedure' with the introduction of the image information at finer resolution. The procedure progresses until all the information has been taken into account. The proposed algorithm has been tested on a variety of real images such as human faces, natural scenes and medical images.

\subsection{Summary}

To summarize, our main accomplishments are detailed as follows:

1. We have for the first time provided a rigorous foundation with respect to which visual estimation and control problems can be studied. We have derived explicit observability criterion to check perspective observability of a dynamical system. We have also derived explicit identifiability results to describe parameters that can be identified. Partial results have been obtained to obtain these parameters using an observer or an identifier.

2. We have provided a new nonlinear estimation and control scheme for the purpose of controlling a robot arm. The exact role of estimation and control using a CCD camera has been detailed that would lead to a new technology viz. Dynamic Visually Guided Control Systems.

3. We have begun to study Active Vision, wherein the camera would be controlled actively resulting in visual attention and where the visual cognition would be controlled using a multiresolution framework and wherein the camera parameters would be automatically adjusted to automatically select the appropriate resolution.

In the coming two years of the proposed research, the active vision framework would be worked out in considerable details.

\section{DISCLAIMER}

This report was prepared as an account of work sponsored by an agency of the United States Government. Neither the United States Government nor any agency thereof, nor any of their employees, makes any warranty, express or implied, or assumes any legal liability or responsibility for the accuracy, completeness, or usefulness of any information, apparatus, product, or process disclosed, or represents that its use would not infringe privately owned rights. Reference herein to any specific commercial product, process, or service by trade name, trademark, manufacturer, or otherwise does not necessarily constitute or imply its endorsement, recommendation, or favoring by the United States Government or any agency thereof. The views and opinions of authors expressed herein do not necessarily state or reflect those of the United States Government or any agency thereof. 


\section{List of PhD students graduated or about to gradu- ate}

1. Y.T. Wu: Some New Problems in Perspective System Theory, 1991.

2. M. Jankovic: Observer Design and Identification of Nonlinear Systems, 1992.

3. M. Lei: Vision Based Robotic Tracking and Manipulation, 1994.

4. E. P. Loucks: A Perspective Systems Approach to Motion and Shape Estimation Problems in Machine Vision, 1994. (in preparation)

5. J. Zhou: New Problems in Motion Estimation without Feature Correspondence, 1994. (in preparation)

6. Zhenyu Yu: Some Experimental Results in Image Guided Survoing of a Robotic Manipulator, 1994. (in preparation)

\section{List of Papers}

1. "Simultaneous Coefficient Assignment of Discrete Time, Multi-Input Multi-Output, Linear Time Varying Systems - A New Approach to Compensator Design," SIAM J. Control and Optimization, vol. 31, No. 5, November 1993, pp. 1438-1461. (with P. Bouthellier)

2. "Perspective Problems in System Theory and its Application to Machine Vision," Journal of Mathematical Systems, Estimation and Control, vol. 4, No. 1, (1994), pp. 3-38. (with Y.T. Wu and M. Jankovic)

3. "A Necessary and Sufficient Condition for the Perspective Observability Problem," Systems and Control Letters. (with X. Wang, C. Martin and W.P. Dayawansa) Accepted for publication

4. "A generalized Popov-Belevitch-Hautus Test of Observability," IEEE Trans. Aut. Contr. (with J. Rosenthal) Accepted for publication.

5. "A Perspective Theory for Motion and Shape Estimation in Machine Vision," submitted to SIAM J. Control and Optimization (with E. P. Loucks)

6. "Visually Guided Ranging from Observations of Points, Lines and Curves via an Identifier Based Nonlinear Observer," submitted to Systems and Control Letters. (with M. Jankovic)

7. "A Realization Theory for Perspective Systems with Applications to Parameter Estimation Problems in Machine Vision," submitted to IEEE Trans. Aut. Contr. (with E. P. Loucks) 
8. "Image Representation and Segmentation using Multiresolution Analysis," submitted to IEEE Trans. Pattern Analysis and Machine Intelligence (with J. Zhou and X. Fang)

9. "Visually Guided Robot Tracking and Grasping of a Moving Object: A Multi-Camera Non- Stereo Approach," manuscript under preparation for IEEE Trans. on Systems Man and Cybernetics. (with M. Lei)

10. "Gradient Based Algorithms for Estimation Problems in Machine Vision," manuscript under preparation for Computer Vision, Graphics and Image Procesing (with J. Zhou)

\section{List of Conference Papers and Presentations}

1. "Observability of Perspective Systems: A New Approach to Computer Vision," Computation and Control II, Proceedings of the Second Bozeman Conference, Bozeman, Montana, August 1-7, 1990, K.L. Bowers and J. Lund Editors, Birkhauser, Boston, 1991, pp. 125-134 (with Y.T. Wu).

2. "Observability and Identifiability Problems in Perspective Systems: A New Approach to Computer Vision," SPIE Vol. 1607 Intelligent Robots and Computer Vision X: Algorithms and Techniques (1991), pp. 589-600. (with Y.T. Wu)

3. "Some New Results in Perspective System Theory and its Application to Computer Vision, "Proceedings of the ninth symposium on energy engineering sciences - Fluid and Dynamical Systems, May 13-15, 1991, at the Argonne National Laboratory, Argonne, Illinois, Report No. CONF-9105116, pp. 85-92.

4. "New Geometric Methods in Computing the Motion Parameters of a Rigid Body Using Straight Line Correspondences," Proc. 1992 American Control Conference, Chicago, pp. 1500-1504, June 1992. (with M. Lei)

5. "An adaptive controller for systems with unmeasurable disturbance," Proc. 1992 American Control Conference," Chicago, June 1992. (with W. Lin)

6. "Some problems in perspective system theory and its application to machine vision," IROS'92, 1992 IEEE/RSJ International Conference on Intelligent Robots and Systems, Raleigh, North Carolina, USA, July 7-10, 1992 (with M. Jankovic and Y.T. Wu.)

7. "Dynamical Systems approach to Computer Vision," SIAM Conference on Applications of Dynamical Systems, Salt Lake City, Utah, October 15-19, 1992.

8. "An optical flow based approach for motion and shape parameter estimation in computer vision," Proceedings of the 31st IEEE Conference on Decision and Control, 1992. (with E. P. Loucks and J. Lund)

9. "Visually guided robotic motion tracking" Proceedings of the Thirtieth Annual Allerton Conference on Communication, Control and Computing, September 30-Oct 2, 1992. (with M. Lei) 
10. "On the problem of parameter identification in perspective systems and its application to motion estimation problems in Computer Vision," Computation and Control III, Proceedings of the Third Bozeman Conference, Bozeman, Montana. K.L. Bowers and J. Lund Editors, Birkhauser, Boston, 1993. (with E. P. Loucks)

11. "Estimation of Angular Velocity of a Moving Object Using Line Correspondences," Proceedings of the American Control Conference, San Francisco, June 2-4, 1993. (with M. Lei)

12. "Image Based Estimation Problems in System Theory: Motion and Shape Estimation of a Planar Textured Surface Undergoing a Rigid Flow," Proceedings of the American Control Conference, San Francisco, June 2-4, pp. 1322-1326, 1993. (Invited)

13. "On the Problem of Coefficient Assignment of Discrete Time Multi-Input Multi-Output Linear Time Varying Systems," Proceedings of the American Control Conference, San Francisco, June 2-4, 1993. (Invited)

14. "A Recursive Approach for Coefficient Assignment of Discrete Time MIMO Linear Time Varying Systems," Proceedings of the 12th IFAC World Congress, Sydney, Australia, 19th- 23rd July, 1993. (Invited)

15. "Visionics: A New Vision Guided Estimation of a Dynamical System," Proceedings of the 12th IFAC World Congress, Sydney, Australia, 19th-23rd July, 1993. (Invited)

16. "Some new results in discrete time motion and shape estimation in machine vision," Proceedings of the Tenth International Symposium on the Mathematical Theory of Networks and Systems, Regensburg, August 2-6, 1993. (Invited) (with E. P. Loucks)

17. "On the Realization of Perspective Systems and its Application to Motion and Shape Estimation Problems in Machine Vision," Proceedings of the 32nd IEEE Conference on Decision and Control, San Antonio, Texas, December 15-17, 1993, pp. 1233-1236. (with E. P. Loucks)

18. 23 "Visually Guided Robotic Tracking and Grasping of a Moving Object," Proceedings of the 32nd IEEE Conference on Decision and Control, San Antonio, Texas, December 15-17, 1993, pp. 1604-1609. (with M. Lei)

\section{$5 \quad$ List of Invited Presentations}

1. "Perspective System Theory: A new perspective in machine vision," Invited Presentation at the Center for applied mathematics, University of Notre Dame, Notre Dame, Indiana 46556, April 18th, 1991.

2. "Observability problems in perspective system theory and its application to computer vision," Presented at the 2nd NIU Conference on Linear Algebra, Numerical Linear Algebra, and Applications,Northern Illinois University, DeKalb, Illinois, May 5, 1991. 
3. "Some New Results in Observer Design and its Application to Perspective Systems," Presented at the 9th Symposium on Energy Engineering Sciences, Argonne National Laboratory, Argonne, IL, May 13, 1991.

4. "A survey of simultaneous stabilization problems for linear time invariant systems and linear time varying systems," Presented at the Department of Control Engineering, Tokyo Institute of Technology, Japan, June 12, 1991, and at the Department of Information Sciences, Tokyo Denki University, Hatoyama-Machi, Hikigun, Saitama, Japan 350-03, 24th June, 1991.

5. "Some new perspective on machine vision," Invited presentation at the the Department of Mathematical Engineering and Information Physics, University of Tokyo, Bunkyoku, Tokyo 113, Japan, June 10, 1991, the Department of Mechanical Engineering, Nagoya University, Japan on 14th June, 1991 and at the Department of Information Sciences, Tokyo Denki University, Hatoyama-Machi, Hikigun, Saitama, Japan 350-03, 25th June, 1991.

6. Problems in perspective system theory and its application to correspondence problems in machine vision," Invited presentation at the Beckmann Institute, University of Illinois at Urbana, Champaign. Dec. 18, 1991. Invited presentation at the General Robotics and Active Sensory Perception Laboratory (GRASP), University of Pennsylvania, 3401 Walnut Street, Philadelphia, PA 19104 on Feb. 19, 1992.

7. Perspective Problems and its Application to Computer Vision and System Theory," Invited presentation at the Department of Mechanical Engineering for ComputerControlled Machinery, Faculty of Engineering, Osaka University, 2-1 Yamadaoka, Suita, Osaka 565, Japan, 25th July, 1992.

8. "Algebraic Geometric Methods in the Study of Line Based Correspondence Problems in Computer Vision," Invited presentation at the Department of Mechanical Engineering for Computer-Controlled Machinery, Faculty of Engineering, Osaka University, 2-1 Yamadaoka, Suita, Osaka 565, Japan, 27th July, 1992.

9. "A new nonlinear feedback controller for visually guided robotic motion tracking." Invited presentation at the Department of Mechanical Engineering for Computer- Controlled Machinery, Faculty of Engineering, Osaka University, 2-1 Yamadaoka, Suita, Osaka 565, Japan, 1st August, 1992.

10. "Some New Results in Computer Vision," Invited Presentation at the Third Conference on Computation and Control, Montana State University, Bozeman, Montana, August, 1992.

11. "Some New Problems in Computer Vision and its Connection to Perspective System Theory," Invited Presentation organized by the Department of Electrical Engineering, Indian Institute of Technology, Delhi, India on 5th August, 1993 and by the IEEE Kharagpur Chapter, Indian Institute of Technology, Kharagpur, India on 26th August, 
1993 and by the Centre for Artificial Intelligence and Robotics, Bangalore, India on 1st September, 1993.

12. "On the Problem of Simultaneous Stabilization and Simultaneous Pole Assignment," "On Output Feedback Regulation and Disturbance Decoupling" and "On the Problem of Visually Guided Control of a Robot Arm," Invited Presentation in the Department of Electrical Engineering at Indian Institute of Technology, Kharagpur, India on 12th August, 1993, 17th August, 1993 and 24th August, 1993 respectively.

13. "Visually Guided Ranging from Observations of Points, Lines and Curves via an Identifier Based Nonlinear Observer," Invited Presentation in the Department of Mechanical Engineering at Indian Institute of Technology, Kharagpur, India on 25th August, 1993.

14. "Current Trends in the Field of Systems and Control" and "Visually Guided Control Problems in Robotics," Invited Presentation at the Institute of Armanent Technoligy, Girinagar, Pune, India on 2nd September, 1993 and on 3rd September, 1993 respectively.

15. "Perspective Problems in Systems Thoery and their Applications to Machine Vision, Invited Presentation in the Coordinated Science Lab., University of Illinois at UrbanaChampaign, Urbana, Illinois 61801 on 16th February, 1994.

16. "Perspective System Theory: A new perspective in machine vision," Invited Presentation at the Center for applied mathematics, University of Notre Dame, Notre Dame, Indiana 46556, April 18th, 1991.

17. "Observability problems in perspective system theory and its application to computer vision," Presented at the 2nd NIU Conference on Linear Algebra, Numerical Linear Algebra, and Applications,Northern Illinois University, DeKalb, Illinois, May 5, 1991.

18. "Some New Results in Observer Design and its Application to Perspective Systems," Presented at the 9th Symposium on Energy Engineering Sciences, Argonne National Laboratory, Argonne, IL, May 13, 1991.

19. "A survey of simultaneous stabilization problems for linear time invariant systems and linear time varying systems," Presented at the Department of Control Engineering, Tokyo Institute of Technology, Japan, June 12, 1991, and at the Department of Information Sciences, Tokyo Denki University, Hatoyama-Machi, Hikigun, Saitama, Japan 350-03, 24th June, 1991.

20. Some new perspective on machine vision," Invited presentation at the the Department of Mathematical Engineering and Information Physics, University of Tokyo, Bunkyoku, Tokyo 113, Japan, June 10, 1991, the Department of Mechanical Engineering, Nagoya University, Japan on 14th June, 1991 and at the Department of Information Sciences, Tokyo Denki University, Hatoyama-Machi, Hikigun, Saitama, Japan 350-03, 25th June, 1991. 
21. "Problems in perspective system theory and its application to correspondence problems in machine vision," Invited presentation at the Beckmann Institute, University of Illinois at Urbana, Champaign. Dec. 18, 1991. Invited presentation at the General Robotics and Active Sensory Perception Laboratory (GRASP), University of Pennsylvania, 3401 Walnut Street, Philadelphia, PA 12104 on Feb. 19, 1992

22. "Perspective Problems and its Application to Computer Vision and System Theory," Invited presentation at the Department of Mechanical Engineering for ComputerControlled Machinery, Faculty of Engineering, Osaka University, 2-1 Yamadaoka, Suita, Osaka 565, Japan, 25th July, 1992.

23. Algebraic Geometric Methods in the Study of Line Based Correspondence Problems in Computer Vision" Invited presentation at the Department of Mechanical Engineering for Computer-Controlled Machinery, Faculty of Engineering, Osaka University, 2-1 Yamadaoka, Suita, Osaka 565, Japan, 27th July, 1992.

24. "A new nonlinear feedback controller for visually guided robotic motion tracking." Invited presentation at the Department of Mechanical Engineering for Computer- Controlled Machinery, Faculty of Engineering, Osaka University, 2-1 Yamadaoka, Suita, Osaka 565, Japan, 1st August, 1992.

25. "Some New Results in Computer Vision," Invited Presentation at the Third Conference on Computation and Control, Montana State University, Bozeman, Montana, August, 1992.

26. "Some New Problems in Computer Vision and its Connection to Perspective System Theory," Invited Presentation organized by the Department of Electrical Engineering, Indian Institute of Technology, Delhi, India on 5th August, 1993 and by the IEEE Kharagpur Chapter, Indian Institute of Technology, Kharagpur, India on 26th August, 1993 and by the Centre for Artificial Intelligence and Robotics, Bangalore, India on 1st September, 1993.

27. On the Problem of Simultaneous Stabilization and Simultaneous Pole Assignment," "On Output Feedback Regulation and Disturbance Decoupling" and "On the Problem of Visually Guided Control of a Robot Arm," Invited Presentation in the Department of Electrical Engineering at Indian Institute of Technology, Kharagpur, India on 12th August, 1993, 17th August, 1993 and 24th August, 1993 respectively.

28. Visually Guided Ranging from Observations of Points, Lines and Curves via an Identifier Based Nonlinear Observer," Invited Presentation in the Department of Mechanical Engineering at Indian Institute of Technology, Kharagpur, India on 25th August, 1993.

29. Current Trends in the Field of Systems and Control" and "Visually Guided Control Problems in Robotics," Invited Presentation at the Institute of Armanent Technoligy, Girinagar, Pune, India on 2nd September, 1993 and on 3rd September, 1993 respectively. 
30. "Perspective Problems in Systems Thoery and their Applications to Machine Vision," Invited Presentation in the Coordinated Science Lab., University of Illinois at UrbanaChampaign, Urbana, Illinois 61801 on 16th February, 1994. 

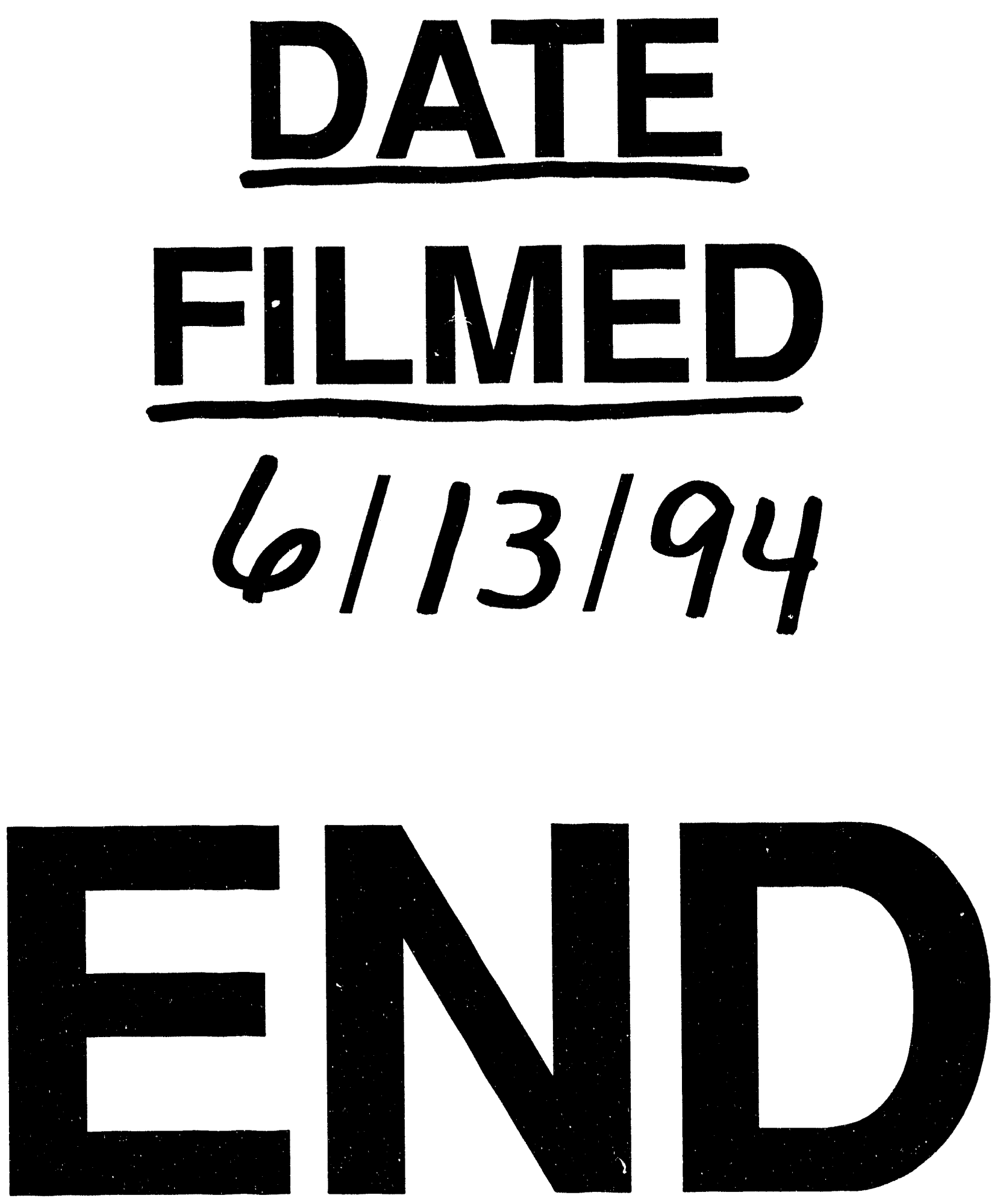